Einführung in das Schwerpunktthema:

\title{
Schlüsseltechnologien und Nachhaltigkeit
}

$\mathrm{D}$ Von Bernd Hirschl und Wilfried Konrad ie Entwicklung neuer Technologien sowie ihre Diffusion im Produktions- und Konsumbereich und die Veränderung ökologischer Zusammenhänge sind eng miteinander gekoppelte Prozesse. Mit der Entstehung der Industriegesellschaften wurden die Natur und ihre Ressourcen der Herrschaft einer technisch-ökonomisch Logik unterworfen. Wachstum und Wohlstand basierten und definierten sich wesentlich über die Ausbeutung natürlicher Ressourcen durch technische Artefakte und Systeme. Die dadurch ausgelösten Zerstörungen führen seit Mitte des letzten Jahrhunderts zur Formierung von Umweltbewegungen, die sich für einen anderen Umgang mit natürlichen Ressourcen einsetzen und dabei größtenteils eine dezidiert technikkritische bis -feindliche Haltung einnahmen.

Seit der Hochzeit der Umweltbewegungen hat der ökologische Skeptizismus gegenüber dem technischen Fortschritt jedoch eine bemerkenswerte Wandlung erfahren. Mittlerweile geht ökologisch motivierte Gesellschaftskritik dazu über, auch nach technologisch bedingten Möglichkeiten für eine Erhaltung und Erneuerung der Umwelt und der Erschließung von Nachhaltigkeitschancen jenseits von Suffizienzparadigmen zu suchen. Beispiele hierfür sind die Nutzung erneuerbarer Energien oder viele Anwendungen der Informationsund Kommunikationstechnik. Auf diesen und anderen sogenannten Schlüsseltechnologien von der Nano- bis zur Biotechnik ruht die große Hoffnung, dass sie neue Wege zu nachhaltigeren Technologiepfaden eröffnen können.

Wie Schlüsseltechnologien politisch eingeordnet werden, welche Herausforderungen mit der Identifikation von Schlüsseltechnologien sowie ihrer Bewertung und Gestaltung unter Nachhaltigkeitsaspekten verbunden sind, was Technologien zu Schlüsseltechnologien macht sind einige der Fragen, die in den folgenden Beiträgen behandelt werden. Sie umfassen sowohl übergreifende Texte als auch solche, die auf spezifische Schlüsseltechnologien fokussieren.

\section{Die Beiträge im Einzelnen}

Der Schwerpunkt wird eröffnet von einem Artikel der Bundesministerin für Bildung und Forschung Edelgard Bulmahn. Sie sieht in der Ausrichtung der Forschungsförderung auf Innovationen einen zentralen Ansatzpunkt für eine Politik, die der Eröffnung gesellschaftlicher und nachhaltiger $\mathrm{Zu}$ kunftsoptionen verpflichtet ist. Dies wird zum einen durch die Förderung der Entwicklung von Schlïsseltechnologien und eine auf die Stärkung der Innovationskompetenz ausgerichtete Bildungspolitik umgesetzt. Zum anderen wird die frühzeitige Identifikation und Beeinflussung von mit Schlüsseltechnologien verbundenen Nachhaltigkeitsrisiken und sozialen Konflikten zur zentralen Aufgabe der Forschungspolitik erklärt. Hier spielen Methoden der Innovations- und Technikanalyse und der Förderschwerpunkt ,,Sozial-ökologische Forschung“ eine zentrale Rolle. Beide sollen das Wissen generieren, die die Gestaltung gesellschaftlicher und technologischer Prozesse im Rahmen nachhaltiger Entwicklung ermöglicht.

Im Anschluss konstatiert Armin Grunwald ein ambivalentes Verhältnis von Nachhaltigkeit und Schlüsseltechnologien. Schlüsseltechnologien von sich aus weder nachhaltig noch nicht nachhaltig; vielmehr wird ihre Nachhaltigkeitswirkung erst im Gesamtzusammenhang von Herstellung, Nutzung und Entsorgung sowie ihrer gesellschaftlichen Einbettung bestimmt. Die Bewertung und Gestaltung von Schlüsseltechnologien unter Nachhaltigkeitsaspekten ist daher als begleitender, reflexiver Lernprozess zu realisieren.

Aufgrund der großen Zukunftsrelevanz von Schlüsseltechnologien geht Axel Zweck davon aus, dass die frühzeitige Erkennung von Schlüsseltechnologien von hohem gesellschaftlich Interesse sind. Als ein geeignetes Instrument zur Identifikation von Schlüsseltechnologien wird die Technologiefrüherkennung vorgestellt. Darüber hinaus wird der besondere Stellenwert partizipativer Verfahren betont, mit denen gesellschaftliche Anforderungen in die Diskussion um zukünftige Schlüsseltechnologien einbezogen werden können.
Die nachfolgenden Artikel wenden sich konkreten Schlüsseltechnologien zu.

Bernd Hirschl und Esther Hoffmann fokussieren auf die Brennstoffzelle - eine Technologie, die über ihre Anwendung im Auto selbst in der Grünen Partei große Sympathie für diese Art der mobilen Fortbewegung erzeugt hat. Zur potenziellen Schlüsseltechnologie wird die Brennstoffzelle durch die Einsatzvielfalt sowie durch ihre Vielseitigkeit bezüglich der Brennstoffe. Die AutorInnen betonen, dass in der Brennstoffwahl der entscheidende Schlüssel der Nachhaltigkeitsbewertung liegt.

Michael Steinfeldt beschreibt den Kontext der Nachhaltigkeit für den Bereich der Nanotechnologie auf der Basis eines derzeit laufenden Forschungsvorhabens. Er fokussiert dabei auf einen prospektiven Technologiebewertungs- und Gestaltungsansatz, der zur Versachlichung der Diskussion über Nanotechnologie beitragen kann.

Cornelia R. Karger widmet sich dem Feld der Biotechnologie. Ein konstituierendes Merkmal der Diskussionen über Biotechnologie ist die Unsicherheit über ihre möglichen Wirkungen und Risiken. Als einen Weg für den systematischen Umgang mit Unsicherheit wählte die Autorin innerhalb ihrer Arbeit den Ansatz der Unsicherheitsanalyse. Im Rahmen des Beitrags werden der Ansatz sowie Ergebnisse eines spezifischen Anwendungsfalls beschrieben.

Den Abschluss des Schwerpunktes bildet der Beitrag von Stefan Zundel, der den Konsequenzen der Unausweichlichkeit des technischen Fortschritts nachgeht. Einerseits wird bezweifelt, dass mit einer auf die Förderung von Schlüsseltechnologien ausgerichteten Technologiepolitik kapitalistische Wachstumsschwächen behoben werden können. Andererseits wird darauf hingewiesen, dass die Innovationsdynamik mit Blick etwa auf ihre künftigen ökologischen Chancen und Risiken gesellschaftlich beeinflusst werden muss.

\section{Die Autoren}

Bernd Hirschl und Dr. Wilfried Konrad sind wissenschaftliche Mitarbeiter des Forschungsfeldes Ökologische Produktpolitik am Institut für ökologische Wirtschaftsforschung (IÖW).

Kontakt: Bernd Hirschl, IÖW, Potsdamer Str. 105, 10785 Berlin. Tel. 030-88459426,

E-Mail: bernd.hirschl@ioew.de.

Dr. Wilfried Konrad, IÖW, Büro Heidelberg, Bergstr. 7, 69120 Heidelberg. Tel. 06221-649165, E-Mail: wilfried.konrad@heidelberg.ioew.de 
(c) 20I0 Authors; licensee IÖW and oekom verlag. This is an article distributed under the terms of the Creative Commons Attribution Non-Commercial No Derivates License (http://creativecommons.org/licenses/by-nc-nd/3.o/), which permits unrestricted use, distribution, and reproduction in any medium, provided the original work is properly cited. 